\title{
Comparison of infrared thermography modelling approaches for detection of defect areas in electronic structures
}

\author{
by A. Stoynova and B. Bonev
}

Technical University of Sofia, 8, Kliment Ohridski Blvd., Sofia, Bulgaria, ava@ecad.tu-sofia.bg

\begin{abstract}
The article presents an approach to obtain solutions by channelling the electro-thermal (ET) analogy and using an electrical circuit simulator to model active infrared thermography in non-destructive testing of electronic structures. The analogy can be used to determinate an optimal thermographic procedure parameters to effectively detect hidden defects and malfunctions in electronic devices and structures. To evaluate the effectiveness of the approach, the results of the FEM, FDM, and ET modelling of different regimes of active thermography for non-destructive testing of electronic structures have been compared and verified by experimental measurements.
\end{abstract}

\section{Introduction}

Temperature is one of the most important factors for reliability and hence the quality of electronic devices and circuits [1]. In this aspect, thermal testing and thermal diagnostics are some of the most informative tools for characterizing health, predicting faults and assessing the life of this type of products. Infrared NDE technology provides ability to detect failures and mitigate risks in electronic devices and circuits. The analysis based on electrical modelling of heat transfer is popular and widely used to study the thermal behaviour of electronic devices [2] and infrared thermography modelling [3, 4]. The electro-thermal analogy is also used to model defects in materials [5].

The electro-thermal method of temperature simulation using equivalent RC models is much easier and "intuitive" for designers of the electronic devices and equipment. Defective area or failure location in the electronic structures can be monitoring by applying electrical excitation (pulsed, stepped or modulated power supply or input electrical signal) to perform pulse, step or lock-in thermographic mode of active thermography, respectively.

The evaluation of defect parameters and the material properties of electronic structures is an important aspect of the overall defect assessment process. Estimating these parameters from the heat profile requires a reverse approach. In this sense, a study and evaluation of the trend of surface temperature change was performed to estimate the depth of defects in different electronic structures, comparing the results from digital models of active thermography based on FEM, FDM and ET.

\section{Experimental}

Experimental samples of electronic structures with artificial defects have been prepared and 1D, 2D and 3D electro-thermal models of pulse, step and lock-in thermography have been created with circuit simulation program (LTspice). One 1D pulse thermography model is illustrated on Fig.1a and the model parameters are shown on Fig. 1b.

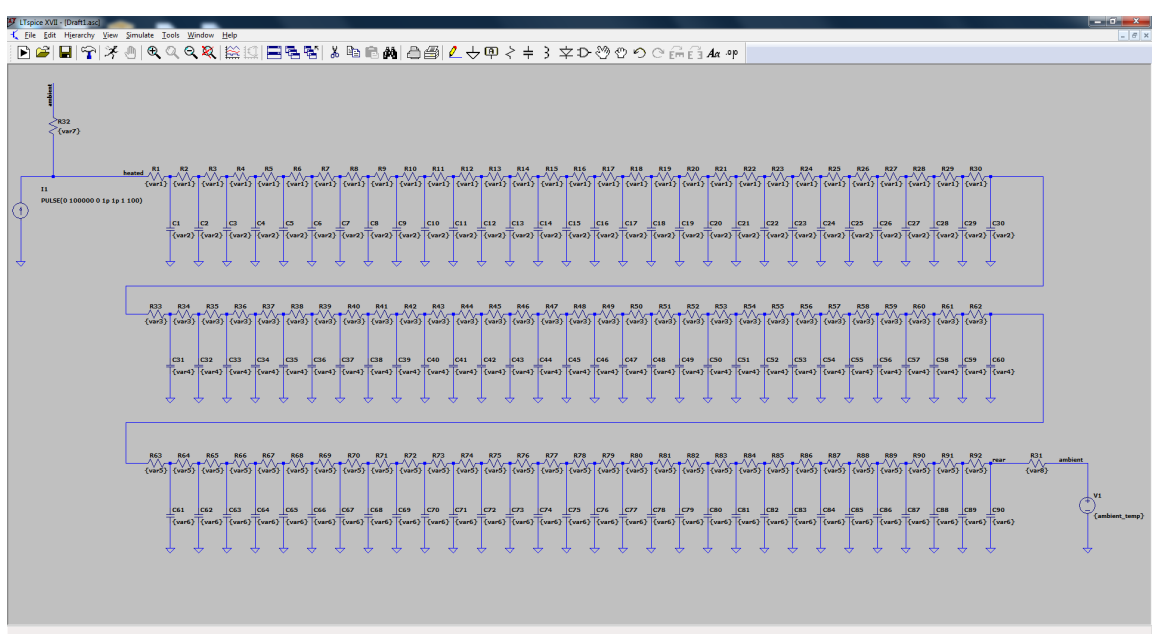

(a)

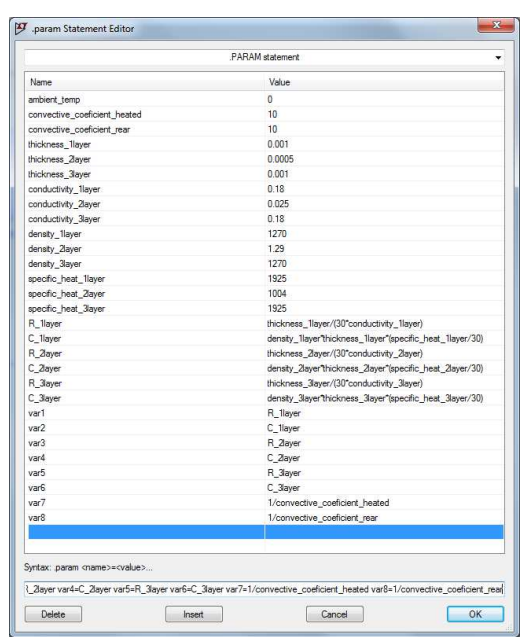

(b)

Fig. 1. (a) Electro-thermal model of defect area in the LTspice program; (b) Parameters of the model 
FEM (finite element method) and FDM (finite difference method) models have been created for different active thermography procedures. Fig. 2 shows the temperature profiles for 1D analytical modeling (Layer 3 Analytic), digital modeling (ThermoCalc3D) and thermo-electric modeling (LTspice). The output parameters of the model are the same as for Fig. 1.

To quantify defects an evaluate indicators are selected as temperature differences and contrasts.

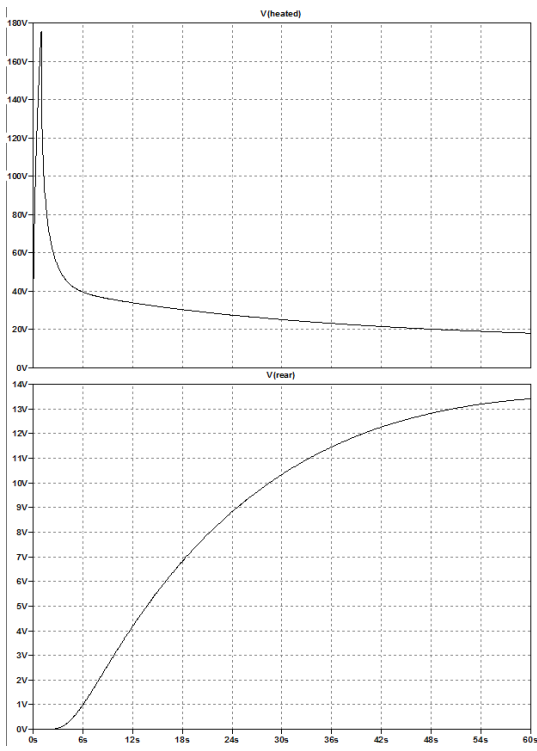

(a) LTSpice
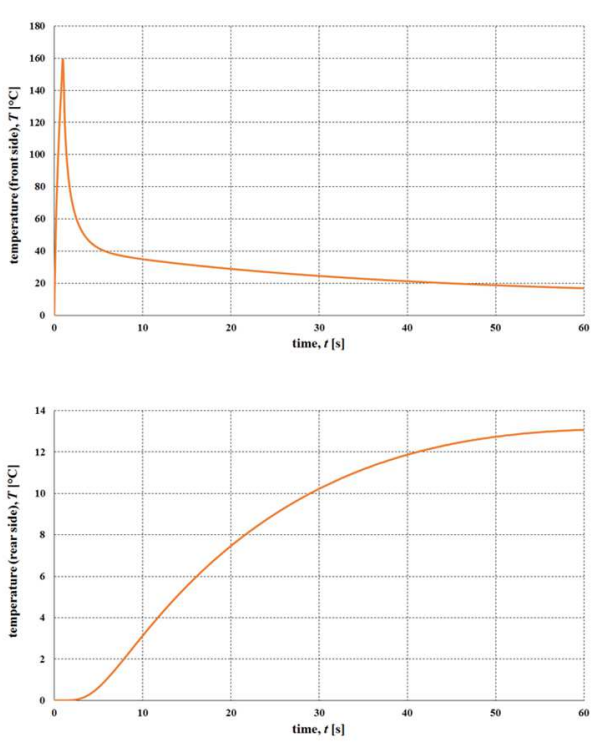

(b) ThermoCalc3D
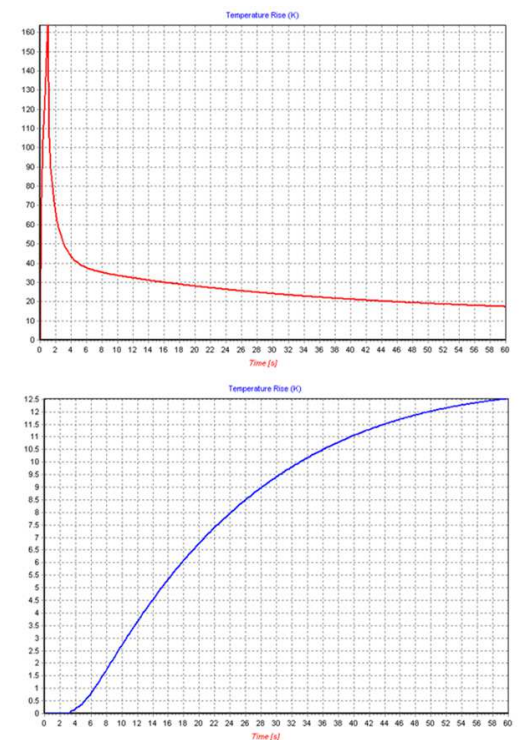

(c) Layer 3 Analytic

Fig. 2. Temperature profiles of flash thermography for the parameters used in Fig.1

The results of the different models are compared with experimental measurements. Comparison of the different models is performed for optimized thermographic procedures and taking into account the duration of the calculation procedure.

\section{Results}

Electro-thermal models have been used to optimize the parameters of non-destructive (pulsed, stepped and lockin) thermographic procedures for detecting hidden defects and faults in specially designed electronic structures. The developed 1D, 2D and 3D ET models have been compared with FEM, FDM models and conclusions have been drawn about the advantages and disadvantages of electro-thermal modelling of active thermography for the study of electronic structures.

Acknowledgment: The author would like to thank for the support of National Science Fund under which Project No. DN 17/16 the present work was conducted. The authors would like to thank to prof. V. Vavilov, Tomsk polytechnic university, Russia for the support and assistance in thermal modeling and simulation using ThermoCalc 3D and Layer 3 Analytic software.

\section{REFERENCES}

[1] Singla S., "Thermal and Humidity Effects on Electronic Equipment", August 15, https://electronicsforu.com/electronics-projects/electronics-design-guides/thermal-humidity-effect-electronicequip/5, 2018.

[2] Blad G., Klepacki D., Potenck J., "Nonlinear discrete elements in electro-thermal model of thick-film structure", 28th International Spring Seminar on Electronics Technology, pp. 245-250, 2005.

[3] Gupta R., Tuli S., "Heat pulse optimization by SPICE simulation for transient thermography in silicon", inProceedings of SPIE 4710, Thermosense XXIV, pp. 649-656, 2002.

[4] Mulaveesala R., Tuli S., "Electro-thermal modeling and matlab-simulink simulation of lock-in thermography for non-destructive characterisation", https://www.ndt.net/article/wcndt2004/html/htmltxt/354_mula.htm, 2004.

[5] Jena P., Mohammed H., Kumar S., Gupta R., "Application of Electro-thermal Approach for Non-Destructive Evaluation of Materials and Structures by Infrared Thermography", Journal of Non Destructive Testing \& Evaluation, pp. 22-29, www.isnt.org.in, March 2018.

[6] Vavilov V., "Modelling Thermal NDT Problems", https://www.ndt.net/search/docs.php3?id=12809, 2012. 